\title{
Cytotoxicity of a novel nano-silver particle endodontic irrigant
}

This article was published in the following Dove Press journal:

Clinical, Cosmetic and Investigational Dentistry

13 July 2015

Number of times this article has been viewed

\section{Eric LK Chan' \\ Chengfei Zhang ${ }^{2}$ \\ Gary SP Cheung ${ }^{2}$}

'Department of Health, Government of Hong Kong SAR, Hong Kong, Special Administrative Region; ${ }^{2}$ Comprehensive Dental Care (Endodontics), Faculty of Dentistry, University of Hong Kong, Hong Kong Special Administrative Region of the People's Republic of China
Correspondence: Gary SP Cheung Advanced Endodontics Centre, 6/F, Prince Philip Dental Hospital, 34 Hospital Road, Saiyingpun, Hong Kong, Special Administrative Region of the People's Republic of China

Tel +85228590288

Fax +85225170544

Email spcheung@hku.hk
Purpose: The aim of this study was to evaluate the cytotoxic effect of a novel nano-silver particle $(25.2 \pm 6.5 \mathrm{~nm})$ endodontic irrigant $(0.2 \mathrm{mM})$ and compare it with $3 \%$ sodium hypochlorite.

Materials and methods: Two cell types, mouse fibroblast National Institutes of Health 3T3 (NIH 3T3) and primary human periodontal ligament stem cell (hPDLSCs) were used in a test for the effect of direct and indirect (by separating the agent and cell with a layer of agar) exposure to the two solutions. In the direct exposure experiment, ten groups of cell cultures were exposed to one dilution $(3: 1,2: 1,1: 1,1: 2,1: 3,1: 4,1: 5,1: 6$ or 1:7) of a nano-silver irrigant for 48 hours; the concentration-response function was estimated by determining the number of viable cells in each group by 3-(4,5-dimethylthiazol-2-yl)-2,5-diphenyltetrazolium bromide (MTT) assay. The 50\% lethal dose of the testing irrigant for NIH3T3 and hPDLSCs were estimated. In the second part of the experiment, a modified agar overlaying technique was applied. Twelve culture wells (6-well plate) were divided into three groups $(n=4)$. The cell lysis zone (cytotoxic range) created by the stock nano-silver solution, 3\% sodium hypochlorite, and an isotonic phosphate buffering saline (control) was measured by two double blinded observers (Kappa score $=100 \%$ ). The cytotoxic score of specific irrigant was derived by modified Sjögren's method.

Results: The 50\% lethal doses of the testing nano silver irrigant for NIH $3 \mathrm{~T} 3$ and hPDLSCs after 48 hours of direct exposure were 0.58 and 0.608 dilution of stock solution, respectively. The cytotoxic scores of nano-silver irrigant and control (phosphate buffered saline) on NIH $3 \mathrm{~T} 3$ were $0.25(95 \%$ confidence interval $[\mathrm{CI}]=0$ to 1.04$)$ and $0(95 \% \mathrm{CI}=0$ to 0$)$; and on hPDLSCs were 0.13 (95\% CI $=0$ to 0.52$)$ and 0.25 (95\% CI $=0$ to 1.04$)$, respectively. Toxicity of the test and control group on both mouse fibroblasts $(P>0.05)$ and hPDLSCs $(P=1.00)$ was not statistically different.

Conclusion: Our results showed that the nano-silver irrigant was non-cytotoxic to both NIH $3 \mathrm{~T} 3$ and hPDLSCs.

Keywords: root canal irrigant, biocompatibility, hPDLSC, agar overlay, MTT

\section{Introduction}

The main etiology of apical periodontitis, one of the most common pathologies of endodontic origin, is bacterial infection of dentin and the root canal system. ${ }^{1}$ Success of endodontic treatment does not only depend on elimination of bacteria from the root canal system, but also on prevention of re-infection of the root canal space. ${ }^{2}$

Traditionally, instrumentation of root canal was considered of primary importance in endodontic treatment. ${ }^{3}$ It is worth stressing that the mechanical instrumentation technique, be it by hand or rotary instruments, only debrides $70 \%$ of the radicular space. ${ }^{4}$ It leads to the current philosophy that the main goals of instrumentation are to 
facilitate effective irrigation of the canal space, and to provide a convenient and resistant form for obturation. ${ }^{5}$ Chemical disinfection plays an important role in root canal treatment when management of the etiological factor is concerned. While irrigation plays a role in mechanical washing, lubrication, and dissolution of remaining pulp tissue, the most important function is killing the microbes. ${ }^{6}$

Although various irrigants are available, none of them fulfill all of the ideal properties of an endodontic irrigant. Sodium hypochlorite is a common irrigant due to its potent antibacterial and organic debris dissolving actions. ${ }^{7,8}$ Hypochlorite has been shown to be effective even against bacteria protected by a biofilm. ${ }^{9}$ Its shortcomings include the unpleasant taste, toxicity, incapability to remove the smear layer completely, and possible deterioration of the (flexural) strength of dentine. ${ }^{10}$ The in vivo antibacterial effectiveness of sodium hypochlorite has also been challenged, as it was shown that sodium hypochlorite could be easily inactivated by organic substances in the root canal such as pulp tissue, dentinal collagen, and microbial by-products. Besides, the capability of effective disinfection of difficult-to-reach areas such as fins, anastomoses, apical delta, and lateral canals was also questionable. ${ }^{11}$

The smear layer is produced during mechanical instrumentation. It is an amorphous mixture of microorganisms, microbial antigens, dentin, and necrotic tissue. ${ }^{10}$ Because hypochlorite irrigation only dissolves the organic matters in the smear layer, purposeful removal of the inorganic components of the smear layer by $17 \%$ ethylenediaminetetraacetic acid (EDTA) for 2 to 3 minutes at the final stage of instrumentation was proposed. ${ }^{12}$ A study has shown that this rinsing protocol provides a cleaner canal wall with open dentinal tubules. ${ }^{12}$ However, deterioration of flexural strength and erosion was observed after this final rinse protocol. ${ }^{13}$

Chlorhexidine digluconate (CHX) is another common endodontic irrigant. It has potent antibacterial properties and has good substantivity. ${ }^{14} \mathrm{CHX}$ possesses similar antibacterial properties as hypochlorite; however, it also may be inactivated by organic matters in the root canal. ${ }^{15}$ Although CHX kills bacteria in the biofilm, it leaves organic debris of biofilm which may negatively affect the sealing of the root filling. Unlike hypochlorite, EDTA did not erode the CHX-irrigated root canal surface. ${ }^{16}$ It was proposed that $2 \% \mathrm{CHX}$ can inhibit the host derived matrix metalloproteinase (MMP) which may be beneficial to the longevity of dentine bonding, ${ }^{17}$ but this hypothesis was questioned recently. ${ }^{18}$

Silver was used as an antiseptic agent before the invention of antibiotics. ${ }^{19}$ With the advancement of nano-technology, nano-silver particles are widely used in the medical industry, including wound dressing, creams, and antibacterial coating of medical devices. ${ }^{20}$ Due to the large surface area-to-volume ratio, the antibacterial effect of nano-sized material is much greater than its bulk solid form. In this connection, antibacterial nano-silver particle solution is considered as a potential endodontic irrigant.

Cytotoxicity assay of dental materials, commonly performed using mouse fibroblasts cell-line as target cells, provides preliminary safety information on the application of nano-silver in mammalian cells. ${ }^{21}$ However, little information is available on the effect of nano-silver on endodontic related tissues. Periodontal ligaments are anatomically related with (the apex of) the root canal system, as extruded irrigant will first come into contact with the periodontal tissues. Therefore, the objective of this study was to compare the cytotoxicity of a novel nano-silver irrigant and sodium hypochlorite on mouse fibroblasts and primary human periodontal ligament stem cells (hPDLSCs) tested with both a direct and indirect mode of contact.

\section{Materials and methods}

A novel nano-silver particle endodontic irrigant was synthesized (Figure 1). The average size of the silver particles was about $25.2 \pm 6.5 \mathrm{~nm}$. The $\mathrm{pH}$ value of the irrigant was measured as 7.4. The concentration of the stock solution was $0.2 \mathrm{mM}$. The solution was stored at $4^{\circ} \mathrm{C}$. Before adding into cell culture, the temperature of the solution was raised

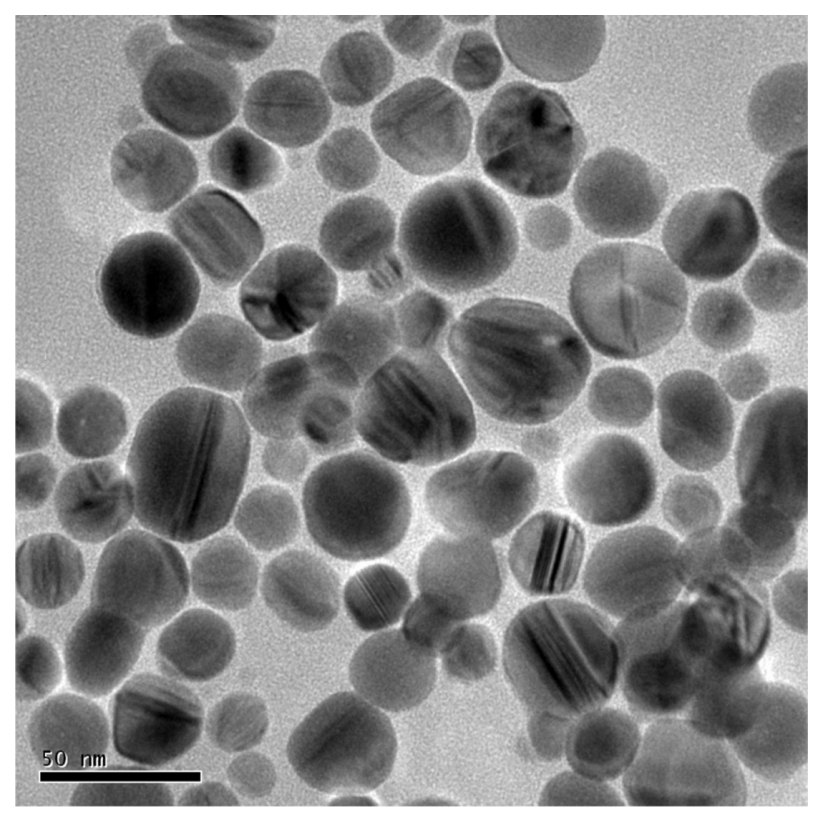

Figure I Transmission electron microscopy image of silver nanoparticles. Notes: The average size of these nanoparticles is about $25.2 \pm 6.5 \mathrm{~nm}$. Courtesy of Prof WT Wong; unpublished data. 
to $37^{\circ} \mathrm{C}$ in a water bath. The cytotoxicity test was done in both direct and indirect contact manner. In the first part of the experiment, the test irrigant was directly added into the cell culture. For the indirect contact mode, the agent was separated from the cell culture with a layer of biocompatible agar. Both experiments were performed on mouse fibroblasts, and then on hPDLSCs.

Mouse fibroblast cell line NIH 3T3 was cultured in Dulbecco's Modified Eagle's Medium (DMEM) with 10\% fetal bovine serum (FBS) and 1\% penicillin/streptomycin in a $\mathrm{CO}_{2}$ incubator. The culture was expanded in $4 \times$ dilution when $80 \%$ confluence was achieved. The sixth and seventh passages, P6 and P7, were used in this experiment. Primary hPDLSCs (passage 2) was isolated and expanded by a method published previously. ${ }^{22}$ The cells were maintained in alpha minimum essential medium ( $\alpha$-MEM) with $10 \%$ FBS and $1 \%$ penicillin/streptomycin. Subculture was performed in $4 \times$ dilution when approximately $80 \%$ confluence was achieved. Passage 5 was used in this study. Nano-silver particles in an aqueous form were prepared in a laboratory of the Polytechnic University of Hong Kong. The particle size was in the range of $25.2 \pm 6.5 \mathrm{~nm}$. Sodium hypochlorite solution was obtained from a dental hospital, which was a 1:1 dilution of a household bleach (Clorox ${ }^{\circledR}$ Regular Bleach; The Clorox Company, Oakland, CA, USA). Another agent, 3-(4,5-dimethylthiazol-2-yl)-2,5-diphenyl tetrazolium bromide (MTT) was purchased from Sigma-Aldrich Co (St Louis, MO, USA).

\section{Direct contact method}

Mouse fibroblasts (NIH 3T3) were seeded on 96-well plates (Corning Incorporated, Corning, NY, USA) in a density of $1 \times 10^{4}$ cells per well. After 24 hours of incubation, the cells formed a confluent monolayer on the base plate of the culture well. The adherence of cells was checked by examining under a phase contrast microscope (TMD Inverted Microscope with Phase Contrast; Nikon Corporation, Tokyo, Japan). Only those wells containing a cell layer that was evenly spread across the base of the well were included in the study.

Stock nano-silver particle suspension, the test irrigant, was serially diluted with concentrated DMEM culture medium (10\% FBS and 1\% penicillin/streptomycin supplements) into nine dilutions $(3: 1,2: 1,1: 1,1: 2,1: 3,1: 4,1: 5$, $1: 6$, and 1:7) (International Organization for Standardization [ISO] 10993-5, 2009).

Cell cultures were divided into ten groups with eight wells per group $(n=8)$. For each group, a fixed volume of diluent $(200 \mu \mathrm{L})$ was added into each individual culture well. One group of eight wells with normal culture medium served as control.

After culturing the cells with the diluent in a $\mathrm{CO}_{2}$ incubator for 48 hours, the percentage of viable cells in each well was estimated by MTT assay. The test culture was incubated with $0.1 \mathrm{~mL}$ MTT solution. After 4 hours of incubation, the MTT solution was carefully pipetted out and the residue was carefully rinsed out with $1 \mathrm{~mL}$ phosphate buffered saline (PBS). Two hundred microlitres of dimethyl sulfoxide was dispensed into every well to lyse the cells and elute their intracellular formazan salt.

After 30 minutes, the optical density (OD) of the formazan-stained dimethyl sulfoxide was measured at 590 $\mathrm{nm}$ in a spectrophotometer (Victor ${ }^{\mathrm{TM}} \mathrm{X} 3$; PerkinElmer Inc., Waltham, MA, USA). The average OD of each group, which represented the relative viability of cells in each group, was normalized with respect to the control group using the following formula:

$$
\text { Relative viability }(\%)=\frac{\text { Mean OD }(\text { test })}{\text { Mean OD }(\text { Control })} \times 100
$$

The experiment was repeated on hPDLSCs cultured with $\alpha$-MEM

\section{Indirect contact method}

In the second part of the experiment, stock nano-silver irrigant and the cell culture were separated with a layer of agar. ${ }^{23}$ The cytotoxicity of the test material was represented by a zone of cell lysis in the center of the culture beneath the test material.

A monolayer of mouse fibroblasts (NIH 3T3) was plated evenly on 6-well plates (Corning Incorporated) with DMEM (10\% FBS and 1\% penicillin/streptomycin supplements). The surface area was approximately $9.5 \mathrm{~cm}^{2}$ and each well contained approximately $6 \times 10^{5}$ cells. After incubation for 24 hours in $5 \% \mathrm{CO}_{2}$ and $37^{\circ} \mathrm{C}$ moist environment, the cell culture had basically formed a $100 \%$ confluent monolayer at the base plate. All culture wells were examined under phase contrast microscope to ensure that $100 \%$ confluency was reached in every well. Any wells with uneven cell distribution or inadequate confluency were excluded from the experiment.

After excluding the unsatisfactory cell culture, the culture medium was exchanged with a fixed volume mixture of DMEM, 1\% low melting point agarose, and 10\% FBS (1 mL per well). The mixture with agarose solidified within minutes, forming a thin layer of agar that was approximately $0.5 \mathrm{~mm}$ thick at the center of the culture well. The agar tended to 


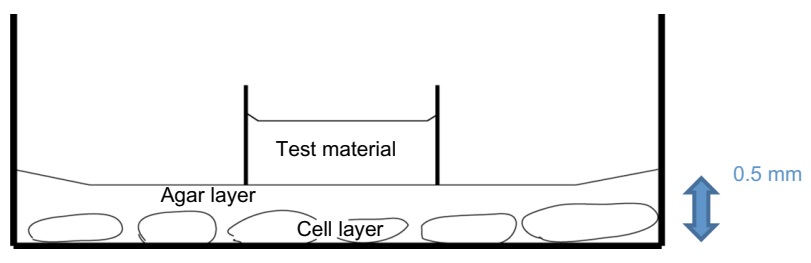

Figure 2 Illustration of the assembly.

Note: A plastic ring was placed on the agar $(0.5 \mathrm{~mm}$ thick) overlaying the cell culture which formed a sample well for the test material.

become thicker at the sides of the culture well due to surface tension (Figure 2).

A sterilized plastic ring was carefully placed on the center of the agar after the initial setting of the agar. The ring was prepared from the plastic lid of a $1.5 \mathrm{~mL}$ centrifuge tube (Corning Incorporated). A seal was secured between the plastic ring and the agar after the agar was completely set (Figure 2).

To confirm the absence of any leakage of the junction between the tube and the agar, about $1 \mathrm{~mL}$ of basal medium was dispensed outside the plastic ring and left for 5 minutes. Those wells with signs of leakage into the ring of sample wells were excluded from the experiment. The wells were divided randomly into three groups (A, B, and C) with four wells per groups $(\mathrm{n}=4)$. Then, $50 \mu \mathrm{L}$ of stock nano-silver irrigant was added into the wells of group $\mathrm{A} ; 50 \mu \mathrm{L}$ of PBS and $3 \%$ sodium hypochlorite was added in the wells of group B and C. After 24 hours of regular incubation, the test solution was rinsed out with $2 \mathrm{~mL}$ of PBS and the plastic rings were removed gently.

One millilitre of the working solution of a cell viability stain (Live/Dead ${ }^{\circledR}$ viability/Cytotoxicity Kit * for mammalian cells*; Thermo Fisher Scientific, Waltham, MA, USA) was added to the cell culture and incubated for 30 minutes. Any changes in cell morphology were detected under a phase contrast microscope in dark field with blue excitation light illumination (495 nm excitation). The fluorescent dye in the viable cell was excited by the blue light $(495 \mathrm{~nm})$ and emitted green light (517 nm) (Figure 3).

Cytotoxicity of each test agent was quantified on the digital micrograph by measuring the diameter of the cell culture that had detached or lysed from the base plate. The measurement was performed by two observers who were blinded to the test material. Before measurement, the definition of the end points of the measurement was explained, assisted by sample photomicrographs. The culture well to be measured was placed on the traveling stage of a phase contrast microscope with the magnification of the objective lens set at $10 \times$, and the diaphragm of the light source was reduced
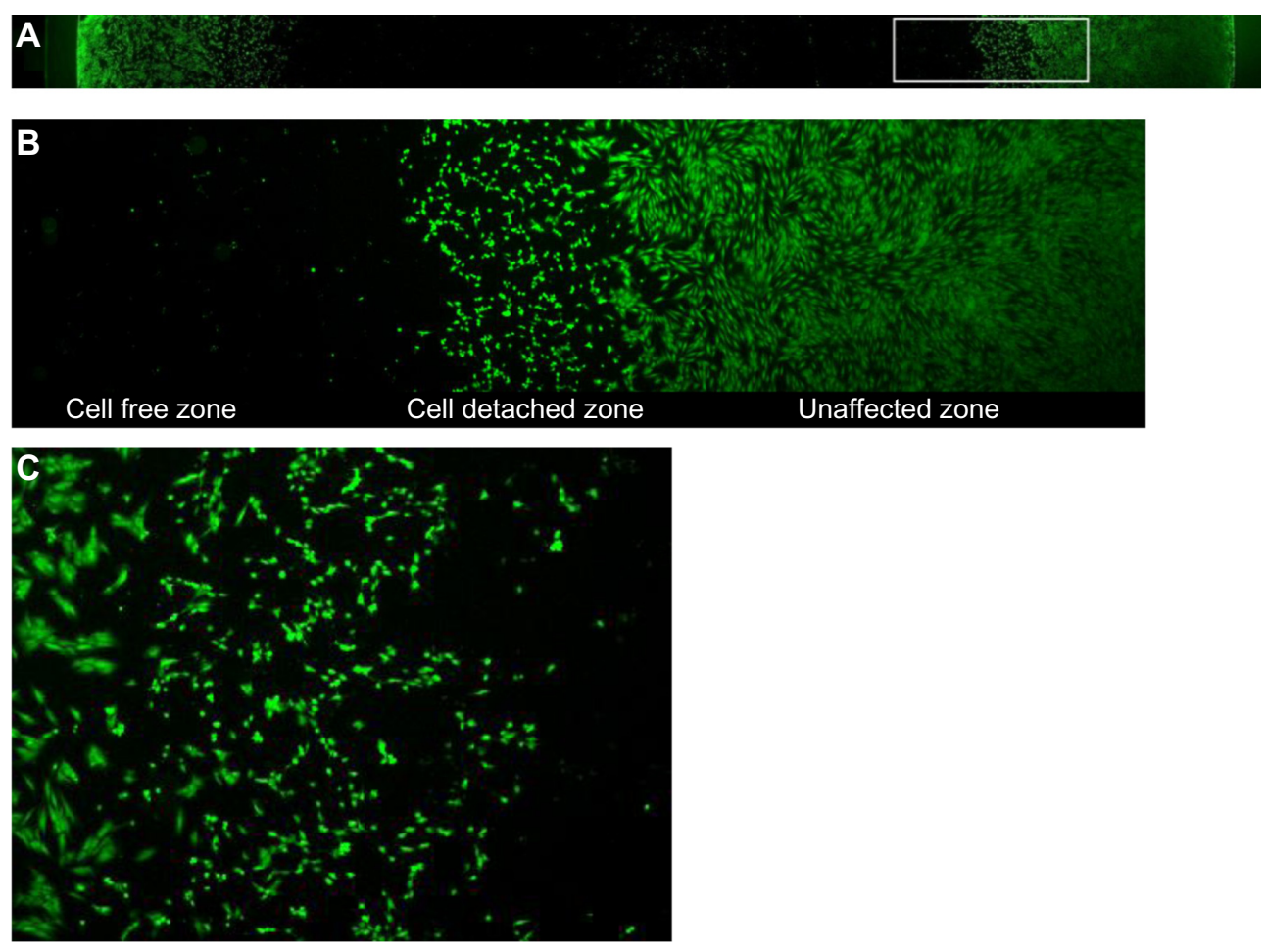

Figure 3 The cytotoxic zone.

Notes: (A) A montage view of live PDL cells (fluorescent green) distributed across the diameter of the well after incubation with $3 \%$ sodium hypochlorite for 24 hours. (B) High-power view showing the boxed area of $(\mathbf{A})$. The transition of cell lysis zone to cell detachment, and then the unaffected zone could be observed. (C) Being affected by the sample solution, the PDL cell was detached from the base plate. The morphology of the cell changed from spindle to oval.

Abbreviation: PDL, periodontal ligament. 
to its smallest size, hence, the field of view was minimized to a small circle of approximately $200 \mu \mathrm{m}$ in diameter at the center of the view (Figure 4).

The stage was moved manually in a defined observation path across the culture well. The start and end points of the observation path were defined as the left and right contact points with the adjacent wells (Figure 5). The locations of where the entire view of the microscope (Figure 4) was covered by adhered cells immediately adjacent to the cell detachment zone were recorded. The diameter of the affected area due to the cytotoxicity of the material, the cytotoxicity range of the sample, was defined as the distance between these two locations.

For each sample well, a cytotoxic score was assigned according to the cytotoxic range as stated in Table 1, with cytotoxic scores derived from modified Sjögren's method. ${ }^{24}$ The median cytotoxic score was calculated from each group and the cytotoxicity of each material was determined and is shown in Table 2.

The experiment was repeated once on hPDLSCs with $\alpha$-MEM.

\section{Statistical analysis}

The Kappa statistic was calculated for the two observers. Analysis of the data was performed by one-way analysis of variance and Bonferroni correction test with SPSS version 17 (SPSS Inc., Chicago, IL, USA).

\section{Results \\ Direct contact}

The results showed a dose-response relationship between the novel nano-silver irrigant with both NIH 3 T3 and

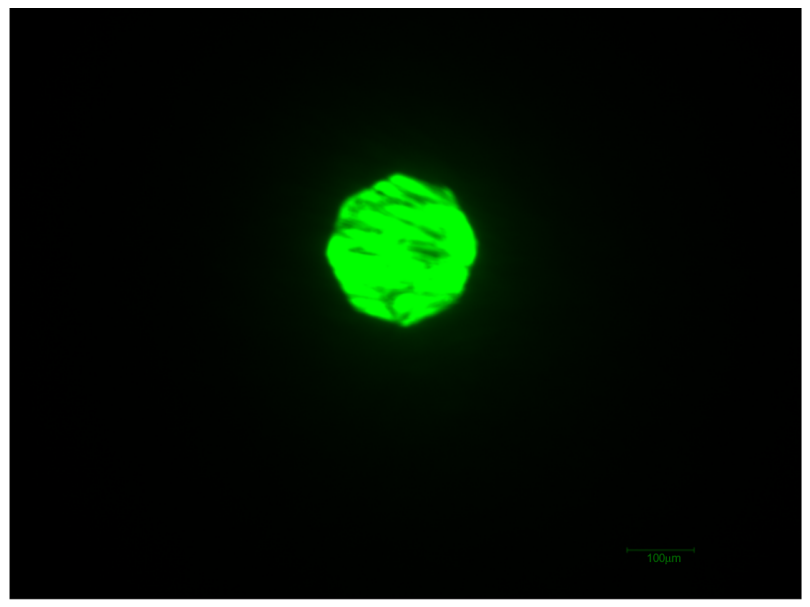

Figure 4 After reducing the diaphragm size of the light source of the phase contrast microscope, at 10× magnification, the field of view covered around I5 PDL cells of about $200 \mu \mathrm{m}$ diameter.

Abbreviation: PDL, periodontal ligament.

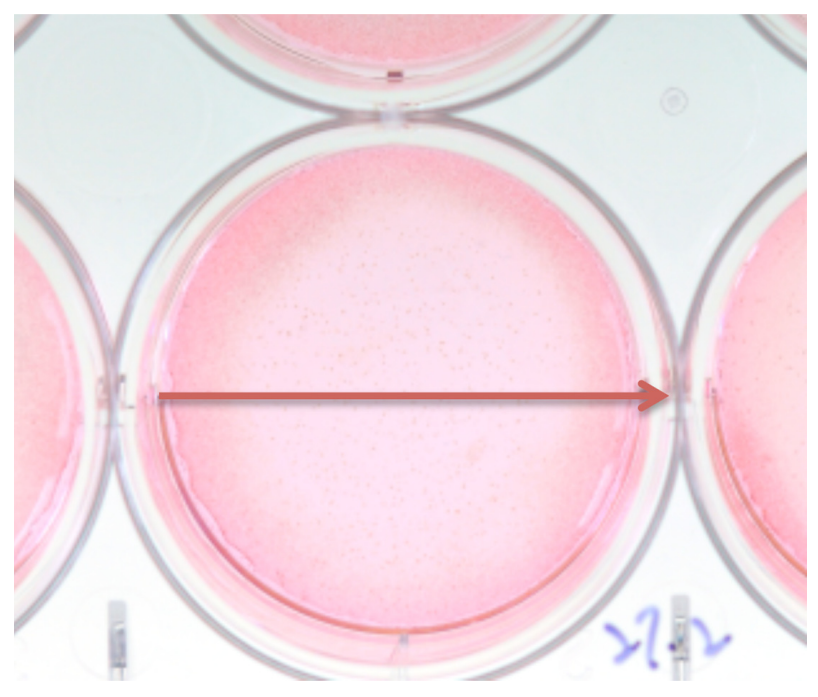

Figure $\mathbf{5}$ The traveling stage of the microscope was traveling along the diameter of the culture well.

hPDLSCs (Figure 6). The stock solution was rather cytotoxic with $97 \%$ of the cells non-viable after direct exposure for 48 hours.

For mouse fibroblast cell line (NIH 3T3), the relationship between the various dilutions and cell viability could be fitted into a cubic regression model:

$$
\begin{aligned}
& \text { Cell viability }(\mathrm{NIH} 3 \mathrm{~T} 3) \\
& \quad=3.277 \mathrm{X}^{3}-5.383 \mathrm{X}^{2}+1.263 \mathrm{X}+0.944\left(R^{2}=0.836\right)
\end{aligned}
$$

where $\mathrm{X}$ represents dilution ratio of the nano-silver irrigant with range 0.00 to 1.00 .

For hPDLSCs, the survival function against the dilution of the irrigant was calculated as:

$$
\begin{aligned}
& \text { Cell viability (hPDLSCs) } \\
& \quad=1.812 \mathrm{X}^{3}-3.159 \mathrm{X}^{2}+0.451 \mathrm{X}+0.993\left(R^{2}=0.871\right)
\end{aligned}
$$

The 50\% lethal dose (LD50) refers to a particular dose (dilution) of the material which causes $50 \%$ of the cells to die

Table I Classification of cytotoxic score

\begin{tabular}{ll}
\hline Cytotoxic score & Cytotoxic range \\
\hline 0 & Cytotoxic range $=0$ \\
1 & Cytotoxic range $\leq 0.6 \mathrm{~cm}$ \\
2 & Cytotoxic range $\leq 1.1 \mathrm{~cm}$ \\
3 & Cytotoxic range $\leq 1.6 \mathrm{~cm}$ \\
4 & Cytotoxic range $>1.6 \mathrm{~cm}$ \\
5 & Whole culture well was affected \\
\hline
\end{tabular}

Notes: For each sample well, a cytotoxic score was assigned according to the cytotoxic range; $(0.6 \mathrm{~cm}$ was the internal diameter of the sample well that contained the test material). 
Table 2 Interpretation of cytotoxic score

\begin{tabular}{ll}
\hline Cytotoxic score & Interpretation \\
\hline $0-0.5$ & Non-cytotoxic \\
$0.6-1.9$ & Mildly cytotoxic \\
$2.0-3.9$ & Moderately cytotoxic \\
$4.0-5.0$ & Markedly cytotoxic \\
\hline
\end{tabular}

Note: The cytotoxic score was classified into four toxicity classes. in the culture within a specific exposure time. Based on the result, the LD50 of the nano-silver solution on mouse fibroblasts NIH 3T3 and hPDLSCs after 48 hours was estimated to be 0.58 and 0.608 dilution ratio, respectively.

Pearson correlation coefficient $(r)$ of data set of both cell types was calculated as $0.884, P<0.001$ (Figure 7), revealing a

A

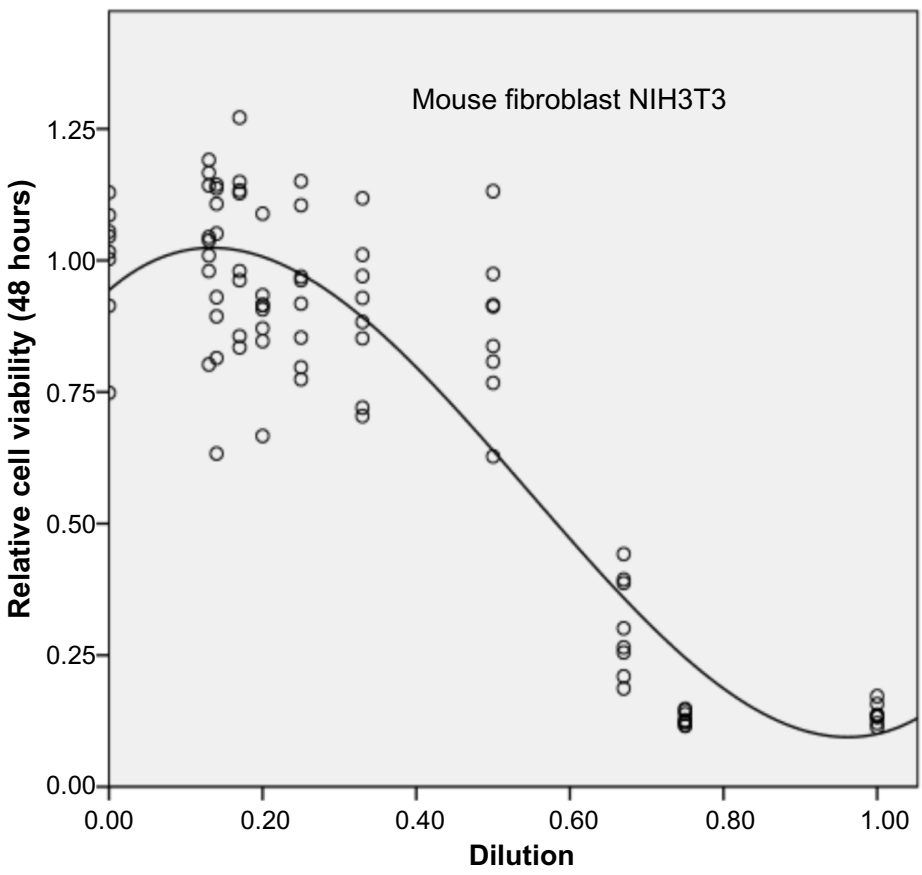

B

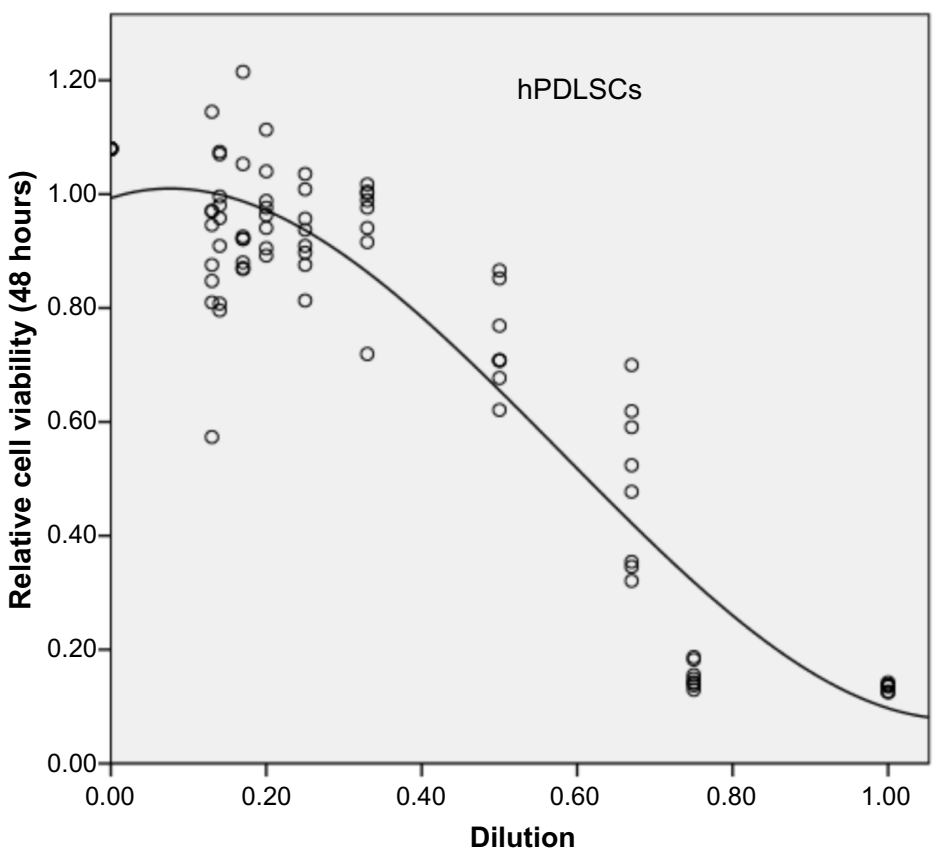

Figure 6 Dose-response relationship between the novel nano-silver irrigant to both NIH 3T3 and hPDLSCs.

Notes: (A) Relative cell viability (48 hours) vs dilution of the stock nano-silver solution on NIH 3 T3 cells. (B) Relative cell viability (48 hours) vs dilution of the stock nanosilver solution on hPDLSCs.

Abbreviations: hPDLSCs, human periodontal ligament stem cells; vs, versus. 


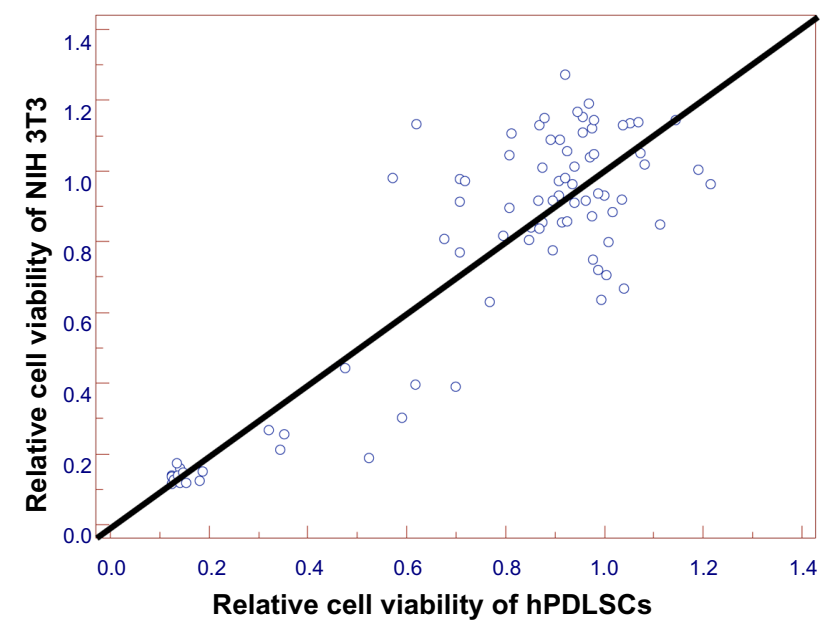

Figure 7 A significant linear relationship of survivability of hPDLSCs and Mouse fibroblast NIH3T3 in different dilutions of nano-silver solution.

Abbreviation: hPDLSCs, human periodontal ligament stem cells.

linear co-relation of dose-response relationship between them in direct contact mode. The dose-response relationship of both cell types appeared to be highly correlated (Figure 8).

\section{Indirect contact}

The inter-observer agreement in this experiment was good, with a Kappa equal to $100 \%$. The responses of mouse fibroblasts (NIH 3T3) and hPDLSCs to the test irrigants were similar. The mean cytotoxic score of nano-silver irrigant and control groups on NIH $3 \mathrm{~T} 3$ was 0.25 (95\% confidence interval [CI] 0-1.04) and 0 (95\% CI 0-0) respectively (Table 3); and there was no statistically significant difference in amount of the test and control groups $(P>0.05)$.

For the hPDLSCs group, the nano-silver solution was classified as non-cytotoxic with a mean cytotoxic score of 0.13 , which was not statistically different from the control group, $0.25(P=1.00)$.

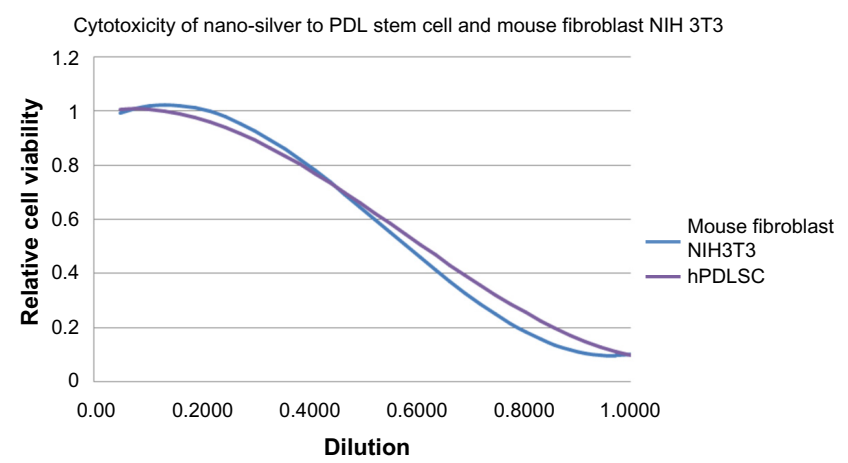

Figure 8 Survival curves (generated by the regression model) of hPDLSCs and Mouse fibroblast NIH3T3 (directly cultured for 48 hours with different dilutions of nano-silver).

Abbreviations: hPDLSCs, human periodontal ligament stem cells; PDL, periodontal ligament.
The 3\% hypochlorite solution exhibited moderate cytotoxicity to both NIH $3 \mathrm{~T} 3$ and hPDLSCs, with mean cytotoxic score of 3.75 (95\% CI 2.95-4.56) and 2.50 (95\% CI 1.58-3.42) respectively, which was significantly more toxic than the control and the nano-silver group to test cells (Table 3).

\section{Discussion}

As recommended by the ISO (10993-5, 2009), if relative cell viability is less than $70 \%$, the test materials would be considered as cytotoxic to that particular cell type. ${ }^{21}$ ISO recommends using NIH 3 T3 or another mouse cell, L929, for cytotoxic screening. Established cell lines are preferred for cytotoxic screening for better reproducibility. However, mouse fibroblast cell lines have been modified genetically for easy culture in a laboratory; cellular response of mouse fibroblast cell lines might differ from human pluripotent cells. The cytotoxicity, genotoxicity, and alteration of differentiation of pluripotent cells might also differ from the animal somatic cells. As the periodontal ligament is in close anatomical proximity to the root apex, information about the effect of endodontic irrigants should be gathered. This experiment focused on the cytotoxicity of a novel irrigant and a commonly used irrigant, sodium hypochlorite (3\%), to both hPDLSCs and mouse fibroblasts NIH 3T3.

Survival of the target cells after direct culturing in serially diluted test solutions was estimated with MTT assay. By regression model, a sigmoidal (cubic) concentrationresponse (survival) function was obtained. The LD50 of the nano-silver particle solution on hPDLSCs and mouse fibroblasts NIH 3T3, was found to be very similar $(0.608$ vs 0.58 times dilution of the stock solution). Linear regression model revealed a linear relationship between the viable cell percentages and the dilution of nano-silver solution for both cell types.

The present methodology followed recommendations of the ISO. ${ }^{21}$ The minimum exposure time of extracts of test material for cytotoxicity assay is 24 hours. In a pilot trial, 24 and 48 hours were compared with the result of the 48 hour group showing a better sigmoidal survival curve. For exposure time limited to 24 hours a relatively steep survival curve was obtained; the LD50 would lie very close to $100 \%$ stock solution in such a case, making the comparison of the curves between different cell types difficult. On the other hand, increasing the exposure time might lead to an overestimation of the cytotoxicity of the material. However, since the silver nanoparticle solution was rated as non-cytotoxic in the present study, the risk of over-estimation is minimal. 
Table 3 Comparison of cytotoxic score of various irrigants on hPDLSCs and Mouse fibroblast NIH3T3

\begin{tabular}{|c|c|c|c|c|c|c|c|c|}
\hline & \multicolumn{4}{|c|}{ Human PDL cells } & \multicolumn{4}{|c|}{ Mouse fibroblast } \\
\hline & \multirow[t]{2}{*}{ Mean } & \multirow[t]{2}{*}{ SD } & \multicolumn{2}{|c|}{ 95\% Cl for Mean } & \multirow[t]{2}{*}{ Mean } & \multirow[t]{2}{*}{ SD } & \multicolumn{2}{|c|}{ 95\% Cl for Mean } \\
\hline & & & Lower bound & Upper bound & & & Lower bound & Upper bound \\
\hline Control & 0.2500 & 0.50000 & 0 & 1.0456 & 0.0000 & 0.00000 & 0.0000 & 0.0000 \\
\hline Nano-silver & 0.1250 & 0.25000 & 0 & 0.5228 & 0.2500 & 0.50000 & 0 & 1.0456 \\
\hline 3\% Нуро & $2.5000^{*, * k}$ & 0.57735 & 1.5813 & 3.4187 & $3.7500^{*, * *}$ & 0.50000 & 2.9544 & 4.5456 \\
\hline
\end{tabular}

Notes: The mean cytotoxic score of hPDLSCs of the $3 \%$ sodium hypochlorite group was significantly higher than control $*(P<0.05)$ and nano-silver group $* *(P<0.05)$. The same comparison was performed on mouse fibroblasts NIH 3 T3. The mean cytotoxic score of NIH 3 T3 of the $3 \%$ hypo group was significantly higher than control $*(P<0.05)$ and nano-silver group $* *(P<0.05)$. The cytotoxic scores of both cell types in various test solutions were compared by crosstab analysis resulting in a $100 \%$ Kappa score.

Abbreviations: hPDLSCs, human periodontal ligament stem cells; PDL, periodontal ligament; hypo, hypochlorite.

For the indirect contact mode, there are two ISO recommended toxicity screening methods, namely, the filter diffusion and the agar overlay method. For the filter diffusion method, the test material is separated from the cell culture with a Millipore filter, which is not suitable when the material is in a liquid form. ${ }^{21}$ In the agar overlay technique, the test material is separated from the cell culture by a layer of agar. ${ }^{23,25}$ There are two basic assumptions behind this method. Firstly, a stable concentration gradient would develop from the well containing the material to the periphery of the culture well; and secondly, the leachate or the test solution would be able to diffuse through the agar layer and to retain its nature upon reaching the target cells. For a sample in liquid form, it was recommended to use biologically inert absorbent filter discs, or making a well at the center of the agar. ${ }^{21}$ In the trial-run of the present study, it was found that only $20 \mu \mathrm{L}$ of test sample could be soaked without seepage. Due to the incubation temperature, some filter discs were dried out after 24 hours. Attempts to make a sample well at the center of the agar were dropped, because that will reduce the agar thickness beneath the well making it quite different from the remaining area, and, technically, it is quite difficult to prepare small wells of a standard depth on a flabby agar sheet. In this present study, standardized plastic tubes were placed to serve as wells for the test solutions. With this method, more solution could be loaded $(50 \mu \mathrm{L})$ and the problem of evaporation during incubation was minimized.

The thickness of the agar might affect the concentration gradient across the culture well. There was no special guideline on the thickness of the agar. In the present study, $0.5 \mathrm{~mm}$ thickness was used. ${ }^{26}$

To evaluate the result, ISO recommended a two-tier approach, one by grading the morphological change of target cells, and another by measuring the size of the affected zone (cytotoxic zone) and categorizing the continuous measurement nominally. ${ }^{21,26}$ To quantify the data objectively and to improve the inter-examiner reliability, only the latter method was used in the present study. It is a common practice to stain the viable cells with neutral red (a vitality stain) to make measurement of the cytotoxic zone possible. ${ }^{21,23} \mathrm{In}$ the pilot study, there was a clear, discolored cytotoxic zone with the mouse fibroblasts NIH 3 T3 cell line that could be measured with the naked eye and a ruler. However, this was not the case in the hPDLSCs group. Although cell lysis was observed under the phase contrast microscope, no visibly discolored zone could be readily identified. A possible explanation might be that the cell density of the culture plate, unlike the cancerous cell line, is lower as a result of contact inhibition. ${ }^{27}$ Thus, these cells tend to grow as a monolayer on the base of the culture well and, since hPDLSCs are much larger than mouse fibroblasts, the cell density would be much lower. As the sensitivity of neutral red vitality staining assay is dependent on a high cell density, the cytotoxic zone in a culture of low cell density might not be well demarcated for naked eye observation. Thus, instead of measurement by ruler and naked eye observation, a more consistent and reproducible method was devised. For this, the neutral red stain was replaced by a fluorescent live/dead stain. Briefly, the stain discriminates live from dead cells by simultaneously staining with green-fluorescent acetomethoxy derivate of calcein to indicate intracellular esterase activity and red-fluorescent ethidium homodimer-1 to indicate loss of plasma membrane integrity. Since the cells were lysed in the cytotoxic zone, staining the dead cells would not be effective in this experiment. Staining only the live cells (green) was done in this experiment. At the margin of the cytotoxic zone, the cell free area and unaffected zone were separated with a cell-detached zone, in which some green stained rounded cells were detached from the culture plate. It is because rounded cells are considered as a morphological sign of cell death, this zone was included in the cytotoxic zone. By crosstab method, the difference of 
survivability of hPDLSCs and NIH3T3 was not statistically significant. This result agreed with the outcome of the direct contact model.

It has been suggested that nano-silver particles may slowly dissolve into a more toxic ionic form. ${ }^{28}$ In this experiment, we did not attempt to control the natural dissolution of silver, because the process of silver dissolution can take months and hence was not expected to have significant impact within 48 hours..$^{29}$ However, further investigation is required regarding any possible silver dissolution and its effect on the long-term safety of nano-silver particle endodontic irrigants to both the human subject and the environment.

\section{Conclusion}

In conclusion, the novel silver nanoparticle irrigant was not cytotoxic to either hPDLSCs or NIH 3T3s in both direct contact and indirect contact assay. The survival response of primary hPDLSCs and mouse fibroblasts NIH 3 T3 to exposure to sodium hypochlorite and a novel silver nanoparticle solution was similar.

\section{Acknowledgments}

The authors are indebted to Dr WT Wong, Professor of Chemistry, Hong Kong Polytechnic University, for synthesizing the nano-silver particles for testing in this study, and providing the micrograph in Figure 1. We also gratefully acknowledge the assistance of Mr Raymond Tong in the preparation of laboratory experiments.

\section{Disclosure}

The authors have no conflicts of interest to disclose.

\section{References}

1. Kakehashi S, Stanley HR, Fitzgerald RJ. The Effects of surgical exposures of dental pulps in germ-free and conventional laboratory rats. Oral Surg Oral Med Oral Pathol. 1965;20:340-349.

2. Ray HA, Trope M. Periapical status of endodontically treated teeth in relation to the technical quality of the root filling and coronal restoration. Int Endod J. 1995;28(1):12-18.

3. Grossman LI. Endodntic Practice. 7th ed. Philadelphia: Lea and Febiger; 1970.

4. Peters OA, Schonenberger K, Laib A. Effects of four Ni-Ti preparation techniques on root canal geometry assessed by micro computed tomography. Int Endod J. 2001;34(3):221-230.

5. Schilder H. Cleaning and shaping the root canal. Dent Clin North Am. 1974;18(2):269-296.

6. Haapasalo M, Shen Y, Qian W, Gao Y. Irrigation in endodontics. Dent Clin North Am. 2010;54(2):291-312.

7. Bystom A, Sundqvist G. Bacteriologic evaluation of the effect of 0.5 precent sodium hypochlorite in endodontic therapy. Oral Surg Oral Med Oral Pathol. 1983;55(3):307-312.

8. Bystrom A, Sundqvist GH. The antibacterial action of sodium hypochlorite and EDTA in 60 cases of endodontic therapy. Int Endod $J$. 1985;18(1):35-40.
9. Clegg MS, Vertucci FJ, Walker C, Belanger M, Britto LR. The effect of exposure to irrigant solutions on apical dentine biofilms in vitro. J Endod. 2006;32(5):434-437.

10. Spångberg L, Engstrom B, Langeland K. Biologic effects of dental materials. 3. Toxicity and antimicrobial effect of endodontic antiseptics in vitro. Oral Surg Oral Med Oral Pathol. 1973;36(6):856-871.

11. Haapasalo HK, Siren EK, Waltimo TM, Ørstavik D, Haapasalo MP. Inactivation of local root canal medicaments by dentine: an in vitro study. Int Endod J. 2000;33(2):126-131.

12. Orstavik D, Haapasalo M. Disinfection by endodontic irrigants and dressings of experimentally infected dentinal tubules. Endod Dent Traumatol. 1990;6(4):142-149.

13. Marending M, Luder HU, Brunner TJ, Knecht S, Stark WJ, Zehnder M. Effect of sodium hypochlorite on human root dentine-mechanical, chemical and structural evaluation. Int Endod J. 2007;40(10):786-793.

14. Delany GM, Patterson SS, Miller $\mathrm{CH}$, Newton $\mathrm{CW}$. The effect of chlorhexidine gluconate irrigation on the root canal flora of freshly extracted necrotic teeth. Oral Surg Oral Med Oral Pathol. 1982;53(5):518-523.

15. Khedmat S1, Aligholi M, Sadeghi S. Influence of bovine serum albumin on the antibacterial activity of endodontic irrigants against Enterococcus faecalis. Iran Endod Jl. 2009;4(4):139-143.

16. Zamany A1, Safavi K, Spångberg LS. The effect of chlorhexidine as an endodontic disinfectant. Oral Surg Oral Med Oral Pathol Oral Radiol Endod. 2003;96(5):578-581.

17. Moon PC, Weaver J, Brooks CN. Review of matrix metalloproteinases' effect on the hybrid dentin bond layer stability and chlorhexidine clinical use to prevent bond failure. Open Dent J. 2010;20(4):147-152.

18. Lührs AK, De Munck J, Geurtsen W, Van Meerbeek B. Does inhibition of proteolytic activity improve adhesive luting? Eur J Oral Sci. 2013;121(2):121-131.

19. Wijnhoven SW, Peijnenburg W, Herberts CA, et al. Nano-silver: A review of available data and knowledge gaps in human and environmental risk assessment. Nanotoxicology. 2009;3(2):109-138.

20. You C, Han C, Wang X, et al. The progress of silver nanoparticles in the antibacterial mechanism, clinical application and cytotoxicity. $\mathrm{Mol}$ Biol Rep. 2012;39(9):9193-9201.

21. International Organization for Standardization. Biological Evaluation of Medical Devices-Part 5: Tests for In Vitro cytotoxicity ISO-10993-5; 2009 (en). 3rd ed. International Organization for Standardization; 2009.

22. Park JC, Kim JM, Jung IH, et al. Isolation and characterization of human periodontal ligament (PDL) stem cells (PDLSCs) from the inflamed PDL tissue: in vitro and in vivo evaluations. J Clin Periodontol. 2011;38(8):721-731.

23. Fehn J, Schottler D. Tissue-culture methods for determining biocompatibility. In: Brown SA, editor. Cell-Culture Test methods: A symposium. West Conshohocken: American Society for Testing and Materials (ASTM) International; 1983:19-50.

24. Sjögren G, Sletten G, Dahl J E. Cytotoxicity of dental alloys, metals, and ceramics assessed by millipore filter, agar overlay, and MTT tests. $J$ Prosthet Dent. 2000;84(2):229-236.

25. Mohammad AR, Mincer HH, Younis O, Dillingham E, Siskin M. Cytotoxicity evaluation of root canal sealers by the tissue culture agar overlay technique. Oral Surg Oral Med Oral Pathol. 1978;45(5): 768-773.

26. Niemi L, Hensten-Pettersen A. In vitro cytotoxicity of Ag-Pd-Cu-based casting alloys. J Biomed Mater Res. 1985;19(5):549-561.

27. Mcclatchey AI, Yap AS. Contact inhibition (of proliferation) redux. Curr Opin Cell Biol. 2012;24(5):685-694.

28. Kvitek L, Panacek A, Prucek R, et al. Antibacterial activity and toxicity of silver - nanosilver versus ionic silver. Journal of Physics Conference Series. 2011;304(1):012029.

29. Dobias J, Bernier-Latmani R. Silver release from silver nanoparticles in natural waters. Environ Sci Technol. 2013;47(9):4140-4146. 


\section{Publish your work in this journal}

Clinical, Cosmetic and Investigational Dentistry is an international, peer-reviewed, open access, online journal focusing on the latest clinical and experimental research in dentistry with specific emphasis on cosmetic interventions. Innovative developments in dental materials, techniques and devices that improve outcomes and patient satisfaction and preference will be highlighted. The manuscript management system is completely online and includes a very quick and fair peerreview system, which is all easy to use. Visit http://www.dovepress. com/testimonials.php to read real quotes from published authors.

Submit your manuscript here: http://www.dovepress.com/clinical-cosmetic-and-investigational-dentistry-journal 